\title{
OEXLE, Otto Gerhard, Das Problem der Problemgeschichte (1880-1932)
}

\section{Alexandre Escudier}

\section{OpenEdition}

\section{Journals}

Édition électronique

URL : http://journals.openedition.org/ifha/1242

DOI : $10.4000 /$ ifha. 1242

ISSN : 2198-8943

\section{Éditeur}

IFRA - Institut franco-allemand (sciences historiques et sociales)

\section{Référence électronique}

Alexandre Escudier, «OEXLE, Otto Gerhard, Das Problem der Problemgeschichte (1880-1932) », Revue de I'IFHA [En ligne], Date de recension, mis en ligne le 01 janvier 2001, consulté le 22 septembre 2020. URL : http://journals.openedition.org/ifha/1242 ; DOI : https://doi.org/10.4000/ifha.1242

Ce document a été généré automatiquement le 22 septembre 2020

(CIFHA 


\title{
OEXLE, Otto Gerhard, Das Problem der Problemgeschichte (1880-1932)
}

\author{
Alexandre Escudier
}

Il ne se passe plus aujourd'hui beaucoup de séminaires ou de colloques, il n'est plus de préface ou d'introduction à l'ambition un tant soit peu méthodique où la notion d'« histoire-problème» (Problemgeschichte) n'apparaisse. Beaucoup plus rares sont les textes où ce type d'approche, présenté comme spécifique et novateur, fait l'objet d'une explicitation théorique satisfaisante. Chacun souhaite aujourd'hui peu ou prou cacher cette partie honteuse de la discipline que serait devenue l'histoire des idées (« ce sol usé jusqu'à la misère ", selon la profession d'anathème de L'archéologie du savoir en 1969), mais on hésite bien souvent en revanche à faire ce saut dans l'inconnu que signifierait une analyse des "pratiques discursives". Aussi se rabat-on d'ordinaire sur l'étiquette d'" histoire-problème ", comme par une convention de langue qui à soi seule garantirait, aux yeux du lecteur, qu'on est à la page, qu'on a comme tout un chacun pris ses distances critiques envers les vieilles lunes de l'histoire des idées, sans avoir pour autant succombé aux sirènes somme toute bien absconses de l'histoire des discours. Le débat, en général, en reste là, le consensus et le vague théoriques battant alors leur plein ; on ne sort pas du rang impunément. C'est dire combien les sciences humaines et sociales, et en particulier l'histoire, ont aujourd'hui besoin d'enquêtes historiographiques qui, suscitées le cas échéant par un sentiment actuel d'insatisfaction théorique, viendraient faire le point critique sur telle ou telle question contemporaine de méthode après en avoir minutieusement établi la généalogique historique. Pour chacune de ces disciplines, et pour tous les questionnaires qui leur sont transversaux, il y aurait là assurément le moyen de passer en revue la mémoire vive de leurs acquis et de régulièrement réviser la cartographie de leurs limites. Nul doute qu'un tel travail d'ensemble permettrait aux jeunes chercheurs de mieux s'orienter dans la forêt contemporaine des innombrables "offres " théoriques; nul doute qu'en résulterait pour chacun une distance réflexive fructueuse par rapport aux pratiques héritées, réduisant par là même à la portion congrue modes intellectuelles et déclarations d'intention théorique de convenance. 
Or c'est précisément à cela que nous invite, une nouvelle fois, O.G.O. avec ce douzième volume des Göttinger Gespräche zur Geschichtswissenschaft (une série désormais bien établie) consacré au thème de l'histoire-problème. Quatre contributions s'y trouvent réunies, qu'une double ambition traverse : à la fois théorique (expliciter ce que recouvre précisément la notion de "Problemgeschichte ») et historiographique (rendre opérationnelle cette notion dans des études concrètes de cas toutes rattachées à la question de l'historisme - au sens de Troeltsch - et de la constitution concomitante de l'histoire en « Historische Kulturwissenschaft » entre 1880 et 1932).

Sur près d'une trentaine de pages, O.G.O. ouvre le recueil par une esquisse générale sur les tenants et aboutissants de la notion d'histoire-problème tout d'abord, sur ses conditions d'émergence ensuite autour de 1900 (à travers notamment le programme épistémologique formulé par Max Weber, mais aussi Georg Simmel et quelques autres, via une critique en règle de l'objectivisme historiciste du XIXe s.), sur le sort enfin qui est aujourd'hui fait à ces courants de pensée jadis concurrents. La thèse ici défendue est tout à fait claire. Contre l'alternative ruineuse de Ranke et d'un certain Nietzsche, contre l'impérieuse nécessité qu'il y aurait, hier comme aujourd'hui, à choisir entre l'historisme objectiviste du XIXe s. et le relativisme décisionniste (voire le postmodernisme contemporain), seule la position balancée de Max Weber est à même de nous tirer d'affaire. Il permet en effet - à la fois - d'accepter en toute quiétude la relativité historique fondamentale de tous les registres de l'activité humaine (à l'inclusion de l'état respectif des savoirs et des instances traditionnelles d'énonciation du normatif) et de continuer d'affiner les critères formels via l'observance desquels la science demeure néanmoins possible à l'intérieur de l'espace délibératif, et analytiquement contraignant, de la communauté scientifique du moment (toutes choses qu'on ne saurait réduire à n'être que la résultante de purs rapports de forces circonstanciels). Ainsi, prendre toute la mesure, avec O.G.O., du diagnostic formulé par Weber au début du siècle, c'est faire acte de mémoire à l'égard de l'héritage théorique fort (i.e. criticiste) des sciences humaines modernes et, partant, se donner les moyens de se tenir à équidistance aussi bien de la théorie du reflet objectiviste du XIXe s. (Abbildtheorie) que des sirènes d'un nietzschéisme débridé à la Stefan George (aux effets délétères que l'on sait sous la République de Weimar). Il y va ici en somme de quelques grandes manières concurrentes de penser la nature et le rôle de la scientificité moderne depuis son avènement ; prendre conscience de la généalogie propre à ces quelques options de pensée, en identifier les motifs fondamentaux et les reformulations contemporaines ne saurait avoir qu'un effet bénéfique pour l'ensemble des sciences humaines et sociales.

À cet article introductif font suite trois esquisses historiographiques (qui donnent un bref avant-goût de trois thèses de doctorat dirigées par 0.G.O.). La première, sous la plume de J. HEINßEN, porte sur Julius Langbehn (1851-1907) et en particulier sur le débat qui se trame, à partir de 1890, autour de son ouvrage Rembrandt als Erzieher. Von einem Deutschen. J. Heinßen y prend à rebrousse-poil la plupart des travaux dont nous disposons aujourd'hui sur les courants pessimistes/critiques de la modernité intellectuelle et socio-politique de l'Europe de la fin du XIXe s. (Kulturkritik ; Kulturpessimismus) et reparcourt ainsi l'ensemble du corpus, en termes d'histoireproblème. Il part du principe que " pathologiser » a priori ces courants de pensée ainsi que leurs principaux protagonistes revient à s'interdire d'accéder à une compréhension véritable de la cohérence propre qui les habite (aussi étrange que tout cela nous puisse paraître aujourd'hui). Ce faisant, il en vient à défendre la thèse générale que cet 
antimodernisme pessimiste de la fin du XIXe s. (ainsi que certains de ses avatars ultérieurs) découle essentiellement d'une volonté de rompre radicalement avec les critères de la rationalité et de la scientificité modernes (Entdisziplinierung ; Entphilosophierung) dans le but de recomposer l'unité sensible et sociale d'une prémodernité fantasmée, au besoin historiographiquement réinventée de toutes pièces. Le " pathologique " se retourne ici comme un gant en avant-garde littéraire et historiographique, par quoi J. Heinßen nous invite instamment à ne pas limiter le champ d'investigation de l'histoire aux seules pratiques historiographiques et pensées de l'histoire ancrées dans les milieux universitaires - disciplinarisés - de la modernité.

La seconde esquisse, due à M. HÄNEL, est encore plus directement reliée à la question de l'historisme au sens troeltschien et porte sur le problème général de l'historicité de la philosophie, bref de la quadrature du cercle que constitue l'idée même d'historiser la philosophie (et partant d'en relativiser l'universalité). C'est toute l'historiographie de l'histoire de la philosophie qui est ici en jeu. Le problème n'est pas nouveau, comme Lutz Geldsetzer l'a bien montré dans son étude pionnière Die Philosophie der Philosophiegeschichte im 19. Jahrhundert (Meisenheim/Glan, 1969); il débute dans le sillage de Kant, et notamment en 1791 sous la plume de Karl Leonard Reinhold (1758-1823) revendiquant l'avènement d'une "Philosophie ohne Beinamen ", d'une philosophie tout court, sans sobriquet ni autres spécifications contingentes. M. Hänel reprend ce dossier pour la période allant de Nietzsche à Cassirer en mettant l'accent sur les nombreux débats ayant eu lieu, du tournant du siècle jusque sous la République de Weimar et parfois au-delà, sur le scandale que constituerait pour la philosophie l'« anarchie des systèmes » qu'elle ne cesse d'engendrer, en dépit des atours de l'universel dont elle s'affuble. Face au défi historiciste qui en découle et qui tend à dissoudre le transcendantal dans l'historique, plusieurs options fondamentales se présentent à l'historiographie de la philosophie : soit elle conserve pour fonction essentielle de décrire par le menu des systèmes philosophiques singuliers (doxographie); soit elle retrace l'odyssée de la philosophie comme telle et dégrade par là même les différents philosophes au rang d'instances contingentes d'actualisation de la Philosophie dans l'histoire (théodicée du Concept) ; soit elle s'efforce de reconstituer à travers l'histoire de la philosophie l'ensemble du spectre thématique et notionnel fondamental de la pensée humaine (anthropologie) ; soit au contraire elle rompt résolument avec chacun de ces modèles somme toute traditionnels pour se métamorphoser elle-même en " histoire-problème " (l'idée même de "système » devenant alors un " problème " pour la philosophie, ainsi que les articulations essentielles de ses avatars historiques successifs). C'est à cette dernière variante qu'invitent les héritiers critiques du néokantisme que sont Nicolai Hartmann (1882-1950) et Ernst Cassirer (1874-1945) ; adoptant des positions comparables à l'épistémologie de Weber pour les sciences humaines et sociales, ils recentrent leur pratique philosophique sur l'historisation de tout ce qui, dans les formes de pensée révolues transmises à la postérité, porte la marque des "problèmes » qui se sont posés aux hommes vivant en société ou que les hommes se sont eux-mêmes posés à travers une activité de " connaissance " plus ou moins formalisée en termes hypothético-déductifs. D'une telle historiographie, une conception renouvelée de la "continuité historique " se dégage, qui rompt radicalement avec toute idée d'incarnation de la « chose en soi » - ou de la substance dans l'étendue ou la pensée (celle-ci ne constituant alors que le passage à la conscience de celle-là). Dans ce cadre, la philosophie comme objet d'histoire ne représente plus qu'une « forme symbolique » parmi d'autres, aux caractères formels spécifiques certes, 
mais essentiellement descriptibles suivant les contours qu'aura pris à telle ou telle époque - suivant tel ou tel défi relevé par l'existence humaine - toute actualisation datable du " problème de la connaissance » en général (Erkenntnisproblem). On lira avec beaucoup d'intérêt ces débats sur la légitimité et les dangers d'une « historisation de la raison ", et l'on s'étonnera tout de même qu'à une époque plus rapprochée de nous la volonté de Michel Foucault d'écrire une " histoire de la vérité » (voire une " histoire de la raison ») ait pu susciter tant de remous alors qu'elle ne représente au bout du compte que l'un des derniers maillons d'une longue série historique. L'oubli historiographique aura été, là encore, la condition de possibilité du scandale (et il n'est pas certain que la recherche historique ait grand profit à tirer des aveuglements passionnels durables que le scandale toujours suscite). Car enfin, l'acception du terme chez Marc Bloch ou chez François Furet mise à part, il est peu d'auteurs en France qui soient aussi proches que Foucault du genre historiographique de l'« histoire-problème »- la notion de « problématisation » revenant du reste sans cesse sous sa plume - et les usages parfois outranciers qui sont faits de son œuvre dans le monde anglo-saxon ne sauraient faire ici illusion sur sa proximité, par dessus sa lecture de Nietzsche, avec le dispositif de pensée mis en place par Max Weber (auquel il a maintes fois fait clairement allégeance à la fin de sa vie). Bref, tout dépend de savoir - comme l'écrit $R$. Laube dans ce volume - « sur quel Nietzsche on met l'accent » (p. 145) et, ajouterai-je, quelle lecture de Nietzsche on privilégie, et l'on ne peut sur ce point qu'appeler de ses vœux des études comparatistes qui, conduites en termes d'histoire de la réception et d'homologies de problèmes, seraient à même de faire apparaître identités et différences entre les courants fondamentaux de l'autoréflexivité des sciences modernes - en Europe et ailleurs.

Dans une troisième et dernière étude, R. LAUBE revient sur la question du « perspectivisme » en Allemagne tel qu'il s'est posé à partir de la critique adressée par Nietzsche à l'histoire dans sa Seconde considération intempestive (1874). En portant notamment - à la manière de Hans Blumenberg - une attention particulière aux jeux de métaphores via lesquels les sciences humaines et sociales explicitent alors les apories fondant leur pratique, il montre d'une part comment le problème de la « Perspektivität " a émergé entre environ 1880 et 1930 dans un contexte général de crise culturelle visant à dissoudre aussi bien l'idée de différenciation sociale que celle de scientificité moderne (différenciation disciplinaire) ; dans le même mouvement, il retrace d'autre part comment l'épistémologie élaborée par Max Weber ainsi que l'interrogation fondamentalement nouvelle de Karl Mannheim en termes de sociologie de la connaissance ont représenté - et représentent sans doute encore aujourd'hui - deux tentatives fortes pour transformer l'armature théorique des sciences de la culture en instance réflexive "re-problématisante ", enfin libérée des apories et des aveuglements subséquents au perspectivisme nietzschéen. Pour ce faire, R. Laube revient dans une première partie sur le programme avancé par Weber pour transformer les sciences de la culture en une histoire-problème qui prendrait définitivement acte de l'historicité fondamentale des interrogations historiographiques ainsi que des appareillages conceptuels déployés pour sans cesse reformuler les réponses qu'on leur apporte (cf. la métaphore de l'idéaltype comme " havre de refuge " (Nothafen) filée par Weber contre la croyance d'un nietzschéisme débridé en l'unité restaurée de la " vie » et de la " science " au-delà des médiations toujours imparfaites et provisoires du langage des sciences). Dans un second temps, R. Laube décrit les articulations centrales des " contre-programmes » d'inspiration nietzschéenne formulés contre l'axiome wébérien 
de l'hétérogénéité de l'être et du devoir-être visant à assumer l'évidence de fait du perspectivisme, sans pour autant jeter la rationalité moderne par dessus bord ; il nous rappelle par là même combien des auteurs aussi différents que René König, Hans-Georg Gadamer et bien d'autres encore ont pu succomber à l'injonction nietzschéenne de devoir, sous peine de tôt ou tard sombrer, surmonter le caractère indéfini et abyssal du perspectivisme (i.e. la relativité historique de tous les registres de l'existence humaine) en reconstituant un " horizon unitaire " de sens et de valeurs, figurant la conscience aiguë des dilemmes de la modernité dans la quiétude retrouvée d'une «naïveté seconde ». Dans la troisième puis la quatrième partie de son exposé, R. Laube retrace - sur fond de biographie intellectuelle et politique - la genèse de la sociologie de la connaissance de Karl Mannheim (le terme de "Wissenssoziologie » étant emprunté à Max Scheler à partir de 1925) dans le sillage des débats sur le perspectivisme du début du siècle, mais surtout grâce à une critique en règle des solutions statiques, voire éthérées, données au problème de l'historisme par Ernst Troeltsch et par Max Scheler via respectivement l'idée d'une "synthèse culturelle " chimérique et un idéalisme anhistorique des valeurs. Mettant l'accent sur le rapport de correspondance existant chez Mannheim entre ordre critique de la pensée et drame biographique (rappelons que, contrairement à $\mathrm{G}$. Lukács qui n'hésitera pas à se retrousser les manches comme commissaire du peuple au nom de l'horizon parousiaque du communisme, il choisit de s'exiler de sa Hongrie natale en 1919 puis est chassé de l'Allemagne nazie en 1933), R. Laube analyse comment l'« autorelativisation » constante de la sociologie de la connaissance, définie comme histoire-problème, la destinait à être systématiquement stigmatisée, à l'extrême droite comme à l'extrême gauche, comme le symptôme d'une « culture de la réflexivité » atomisante à abattre. En conclusion enfin, R. Laube revient sur quelques avatars du rapport à Weber et à l'histoire-problème dans la seconde moitié du XXe s. (chez F. Meinecke, H.-G. Gadamer et H.-U. Wehler).

En quatre coups de sonde judicieusement choisis, ce volume fait clairement apparaître, pour des domaines d'objets en apparence fort hétérogènes, comment les sciences humaines et sociales en sont progressivement venues à se thématiser elles-mêmes en termes d'histoire-problème afin de durablement surmonter un moment de crise profonde de la modernité ainsi que le défi nietzschéen subséquent du perspectivisme. De ce type d'approche, on attend avec impatience la transposition à d'autres pays, d'autres époques, d'autres problèmes. Pour l'heure, excusez du peu, c'est l'histoireproblème elle-même comme méthode qui se trouve objectivée sous nos yeux à travers un corpus de sources dont le degré de conscience réflexive ne le cède en rien à notre époque. Aux accents programmatiques, le titre du recueil ne pouvait prendre, on l'aura compris, qu'un tour spéculaire. Quelques auteurs contemporains (U. Muhlack, H.-U. Wehler, D. Baecker, etc.) en prennent au passage pour leur grade ; c'est que de toute évidence, ici comme ailleurs, « la lumière des grands problèmes de la culture » a passé son chemin et que l'histoire de l'histoire, de votre vivant, a tôt fait de vous communiquer votre acte de décès. Libre à chacun de continuer néanmoins à s'en inquiéter, et de lire Max Weber sans vraiment le lire.

Alexandre ESCUDIER 